\title{
Alterstice
}

Revue internationale de la recherche interculturelle

International Journal of Intercultural Research

Revista International de la Investigacion Intercultural

\section{Recueillir les témoignages des enfants soldats. Pour qui ? Pourquoi ? Comment ?}

\section{Sylvie Bodineau}

Volume 1, numéro 2, 2011

Droits humains et témoignages : l'épreuve de la culture

URI : https://id.erudit.org/iderudit/1077603ar

DOI : https://doi.org/10.7202/1077603ar

Aller au sommaire du numéro

Éditeur(s)

Alterstice

ISSN

1923-919X (numérique)

Découvrir la revue

Citer cet article

Bodineau, S. (2011). Recueillir les témoignages des enfants soldats. Pour qui ? Pourquoi ? Comment ? Alterstice, 1(2), 57-67. https://doi.org/10.7202/1077603ar

\section{Résumé de l'article}

$\mathrm{Au}$ milieu des bouleversements du monde actuel, les enfants font figure de victimes particulières. Pourtant, si la vulnérabilité des enfants est une représentation largement partagée au sein de différentes cultures, ses variations et son appréciation le sont moins, d'autant plus lorsque les événements les placent dans des situations inhabituelles. C'est le cas notamment des " enfants soldats ", phénomène qui n'est pas nouveau et dont les réalités sont très diverses. Basées sur un cadre juridique international qui s'est significativement étendu depuis 1996, les interventions de protection des enfants concernés par le phénomène ne sont pas exemptes de tensions. Elles sont révélatrices de la manière dont les différentes visions et réalités culturelles se conjuguent ou s'affrontent. En effet, les intervenants sont issus de groupes d'origines géographiques, mais aussi professionnelles, sociales, économiques, et/ou politiques différentes, chacun portant ses valeurs et ses intérêts propres. Face aux divergences de vision, la voix des enfants devrait alimenter les analyses et orienter les programmes. Dans les faits, les témoignages des enfants sont utilisés de manière variable. À chaque fois, le recueil de la parole et son utilisation posent des dilemmes propres à ce type d'intervention. Cet article propose, au travers principalement de quatre formes d'utilisation des témoignages (poursuite en justice à la Cour pénale internationale, mécanismes de suivi et de communication de l'information des Nations Unies, visite/échange Nord-Sud, témoignages de " proximité »), une exploration des enjeux et des dilemmes posés. 


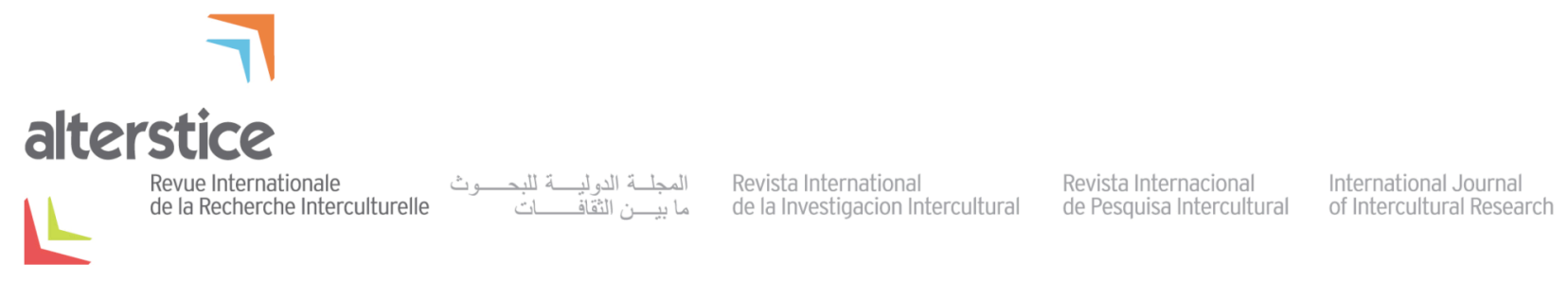

ARTICLE THÉMATIQUE

\section{Recueillir les témoignages des enfants soldats. Pour qui? Pourquoi? Comment?}

Sylvie Bodineau ${ }^{1}$

\section{Résumé}

Au milieu des bouleversements du monde actuel, les enfants font figure de victimes particulières. Pourtant, si la vulnérabilité des enfants est une représentation largement partagée au sein de différentes cultures, ses variations et son appréciation le sont moins, d'autant plus lorsque les événements les placent dans des situations inhabituelles. C'est le cas notamment des " enfants soldats ", phénomène qui n'est pas nouveau et dont les réalités sont très diverses. Basées sur un cadre juridique international qui s'est significativement étendu depuis 1996, les interventions de protection des enfants concernés par le phénomène ne sont pas exemptes de tensions. Elles sont révélatrices de la manière dont les différentes visions et réalités culturelles se conjuguent ou s'affrontent. En effet, les intervenants sont issus de groupes d'origines géographiques, mais aussi professionnelles, sociales, économiques, et/ou politiques différentes, chacun portant ses valeurs et ses intérêts propres. Face aux divergences de vision, la voix des enfants devrait alimenter les analyses et orienter les programmes. Dans les faits, les témoignages des enfants sont utilisés de manière variable. À chaque fois, le recueil de la parole et son utilisation posent des dilemmes propres à ce type d'intervention. Cet article propose, au travers principalement de quatre formes d'utilisation des témoignages (poursuite en justice à la Cour pénale internationale, mécanismes de suivi et de communication de l'information des Nations Unies, visite/échange Nord-Sud, témoignages de " proximité »), une exploration des enjeux et des dilemmes posés.

\section{Rattachement de l'auteure}

${ }^{1}$ Département d'anthropologie et Centre interuniversitaire d’Études sur les Lettres, les Arts et les Traditions (CÉLAT), Université Laval, Québec, Canada

\section{Correspondance}

sylviebodineau@yahoo.fr

\section{Mots clés}

enfants; enfants-soldats; humanitaire; témoignages; protection de l'enfance

\section{Pour citer cet article :}

Bodineau, S. (2011). Recueillir les témoignages des enfants soldats. Pour qui? Pourquoi? Comment? Alterstice, 1(2), 55-66. 


\section{Introduction : utiliser des témoignages d'enfants soldats. Pour qui? Pourquoi? Comment? Dilemmes et tensions autour des figures de l'enfance.}

Les interventions de protection des enfants dans le cadre de l'intervention humanitaire ou de l'aide au développement font l'objet de réflexions et de débats internes, mais aussi de regards et questionnements extérieurs souvent passionnés, quelquefois à caractère manichéen et polarisé dès lors que l'actualité et les médias dirigent leurs projecteurs sur les souffrances des plus «vulnérables ». Ces interventions présentent en effet un certain nombre de tensions, entre discours et pratiques, prétentions et réalisations, espoirs et déceptions, " bonnes » ou « mauvaises » actions, reflets des contradictions de nos sociétés.

Au milieu des bouleversements du monde actuel, les enfants font en effet figure de victimes particulières, d'une part car ils forment une grande partie des réfugiés, déplacés, blessés, affamés, de ceux dont la vie est bouleversée ou rendue difficile en temps de crise et d'autre part parce qu'ils sont considérés comme vulnérables d'emblée, comme en témoigne la Convention relative aux droits de l'enfant (CDE) (Nations Unies, 1989) qui se fonde sur la nécessité de les protéger du fait de leur « immaturité physique et intellectuelle ».

Pourtant, si la vulnérabilité des enfants est une représentation largement partagée au sein de différentes cultures, ses variations et son appréciation le sont beaucoup moins, surtout lorsque les événements les placent dans des situations inhabituelles qui viennent perturber cette représentation de victimes innocentes et passives. En effet, alors qu'ils sont considérés par les acteurs de protection de l'enfance comme en situation de vulnérabilité extrême et faisant l'objet d'interventions de protection, certains enfants se trouvent en position de bourreaux enfants délinquants, trafiquants, membres de gangs - ou très éloignés des figures et positions classiques - enfants prostituées, mères célibataires, enfants sorciers, enfants chefs de famille, etc. C'est aussi le cas des «enfants associés aux forces et groupes armés » plus connus sous l'appellation « d'enfants soldats », appellation qui polarise à elle seule plusieurs représentations fondant un intolérable, puisque, selon certains auteurs, on ne pourrait pas être à la fois enfant (innocent) et soldat (bourreau) (Honwana, 2000; Arzoumanian et Pizzutelli, 2003).

Si le phénomène dit des " enfants soldats" n'est pas nouveau, et même si le discours humanitaire en attribue l'expansion actuelle aux nouvelles formes de guerre et à la prolifération des armes légères et de petit calibre, sa perception du point de vue de l'Occident comme immorale et illégitime l'est, résultat, selon Lee, d'une construction historique récente "shaped by particular social and political forces" (Lee, 2009, p.4). Côté chiffres, certaines estimations annoncent entre 300000 et un million d'enfants - garçons et filles - impliqués dans plus de 30 conflits dans le monde.

Au-delà des chiffres et des images, les réalités sont très diverses et seulement partiellement reflétées par la définition qu'emploient les acteurs de protection de l'enfance pour les qualifier. Selon les agences des Nations Unies et les principales organisations impliquées dans ce secteur d'intervention, un « enfant associé à une force ou un groupe armé » s'entend de

toute personne de moins de 18 ans qui est ou a été recrutée ou utilisée par une force ou un groupe armé, quelle que soit la fonction qu'elle y exerce. Il peut s'agir notamment mais pas exclusivement d'enfants, filles et garçons, utilisés comme combattants, cuisiniers, porteurs, messagers, espions, ou à des fins sexuelles. Le terme ne désigne pas seulement un enfant qui participe ou a participé à des hostilités. (Les Principes de Paris, 2007, § 2.1).

Les interventions de protection qui y sont relatives se sont développées à la suite de la publication en 1996 d'un rapport de Graça Machel commandité par les Nations Unies sur l'impact des conflits armés sur les enfants (Machel, 1996). Elles s'inséraient alors dans un double mouvement. D’une part, l'indignation du monde occidental était grandissante vis-à-vis des pratiques de recrutement d'enfants au sein des forces ou groupes armés (l'enfant est apparu au programme des Nations Unies avec l'adoption de la CDE en 1989). D'autre part, les programmes de Désarmement, Démobilisation et Réinsertion (DDR) soutenus par les missions de maintien de la paix des Nations Unies étaient en expansion. 
Les interventions de protection des enfants soldats sont multiformes, revêtant des aspects à la fois psychosociaux, économiques et géopolitiques complexes, allant du plaidoyer pour leur « libération " à des appuis destinés à favoriser leur réinsertion dans la vie civile. Basées sur un cadre juridique international qui s'est significativement étendu depuis 1996 (en matière de droits humains ${ }^{1}$ mais aussi dans le cadre du Conseil de Sécurité des Nations Unies), elles rencontrent un certain nombre de tensions et de dilemmes révélateurs de la manière dont les différentes visions et réalités culturelles se conjuguent ou s'affrontent. Car ces tensions sont le fruit d'une interculturalité qui n'est pas simplement duelle - faisant se rencontrer une vision " internationale/occidentale liée aux droits humains dits universels » et une vision " locale » de ces enfants - mais aussi plurielle. S'y côtoient en effet des groupes et individus ayant des valeurs et intérêts propres et d'origines géographiques, mais aussi professionnelles, sociales, économiques, et/ou politiques différentes.

Face aux divergences de visions, la voix des enfants concernés - qui sont quand même les ultimes bénéficiaires des interventions de protection - devrait alimenter les analyses et faire partie de toute évaluation de la situation, planification et orientation des interventions dans l'espoir d'une meilleure pertinence et efficacité des programmes. C'est d'ailleurs l'un des principes de la CDE soutenu par l'Article 12, § 1 :

Les États parties garantissent à l'enfant qui est capable de discernement le droit d'exprimer librement son opinion sur toute question l'intéressant, les opinions de l'enfant étant dûment prises en considération eu égard à son âge et à son degré de maturité. (Nations Unies, 1989)

Dans le cadre des programmes relatifs au phénomène des enfants soldats, le recueil et l'utilisation de la parole des enfants ne vont pourtant pas de soi et posent des problèmes propres à ce type d'intervention et à ses contextes, révélant une forme d'interculturalité plurielle autour des différents enjeux et des représentations de l'enfance dans la guerre.

Dans cet article, nous nous penchons sur l'utilisation des témoignages des enfants soldats par les acteurs de protection de l'enfance pour mettre à jour ces dilemmes et ces tensions. Nous avons choisi comme cadre la République Démocratique du Congo (RDC) qui offre une perspective « riche d'expérience » sur le sujet. En effet, parallèlement au mouvement global de dénonciation du phénomène, le recrutement d'enfants soldats est apparu en RDC en 1996 avec la guerre dite "de libération » et se poursuit encore. S’y sont développées un certain nombre de réponses variées correspondant à l'évolution de la thématique des enfants soldats dans la littérature et celle des politiques des organisations humanitaires depuis 1996. Les interventions qui s'y sont déroulées sont indéniablement liées au contexte géopolitique du pays et au processus national formel dit de DDR, ainsi qu'à I'intégration des combattants adultes des différents groupes armés belligérants aux forces armées nationales (FARDC) entre 2004 et 2006, sous les auspices et avec le soutien de la communauté internationale. Enfin, le premier procès de la Cour Pénale Internationale (CPI) pour crime de guerre - crime constitué par le recrutement et l'utilisation d'enfants de moins de 15 ans -, procès où le témoignage des enfants occupe une position centrale, est celui de Thomas Lumbanga, pour des actes commis dans la région de l'Ituri en RDC.

Nous explorons quatre formes d'intervention qui se sont déroulées à différentes périodes et dans lesquelles le témoignage des enfants est central. Pour chacune d'entre elles, nous décrivons le contexte et les faits marquants, puis nous abordons les questionnements qui les traversent, questionnements liés aux enjeux et aux représentations des différents acteurs impliqués. Les quatre situations étudiées sont les suivantes : poursuite en justice à la CPI, mécanismes de suivi et communication de l'information concernant les violations graves des droits de l'enfant suivant les Résolutions du Conseil de Sécurité des Nations Unies n¹539, 1612 et 1882, visite/échange entre enfants du Nord et du Sud et enfin témoignages "de proximité ". Les deux premières situations illustrent le lien entre mécanismes globaux et situations locales. Les deux dernières éclairent plus particulièrement les dynamiques des groupes d'enfants sur un plan transnational et local.

\section{Le cas de témoignage des victimes lors du procès instruit contre Thomas Lubanga Dyilo par la CPI}

Thomas Lubanga, livré à la CPI en 2006, doit y répondre de trois chefs d'accusation pour crimes de guerre, sur la base de sa responsabilité pénale individuelle, pour la conscription et l'enrôlement d'enfants de moins de 15 ans et

\footnotetext{
${ }^{1}$ L'usage de cette expression est expliqué dans le texte introductif au numéro (Saillant, 2011).
} 
pour les avoir fait activement participer à des hostilités (Articles 8(2)(e)(vii) et 25(3)(a) du statut de Rome de la CPI). Les charges alléguées font référence à des crimes ${ }^{2}$ qui ont été commis dans la région de l'Ituri, dans le nord-est de la RDC, crimes dont il n'est pas le seul accusé, cette province étant alors en proie à une violente guerre civile opposant différentes milices, autour notamment du contrôle de secteurs miniers. Deux autres chefs de milice, Germain Katanga et Mathieu Ngudjolo, d'ethnies alliées mais rivales de celle de Lubanga, sont actuellement détenus à La Haye, accusés eux aussi d’avoir utilisé des enfants pour attaquer des civils début 2003.

Fait notable pour ce qui nous intéresse, ce premier procès de la CPI exemplaire pour le choix de la problématique du recrutement et de l'utilisation des enfants est aussi caractérisé par sa primauté quant à l'utilisation du témoignage des enfants. C'est en effet la première fois que des enfants sont appelés à participer et témoigner devant un tribunal international en tant que victimes, avec des modalités de participation spéciales, différentes cependant des systèmes juridiques de droit civil où les victimes ont les mêmes droits que le procureur. Rappelons que, dans le cadre du Tribunal Spécial pour la Sierra Leone qui instruisait des faits d'enlèvement et de recrutement forcé d'enfants de moins de 15 ans dans les forces et groupes armés pour les faire participer à des hostilités, des enfants avaient été appelés à déposer, mais en tant que témoins. Quelques faits marquants du procès contre Thomas Lubanga depuis 2006 nous éclairent sur ce que peuvent être les dilemmes posés par cette nouvelle forme d'utilisation du témoignage des enfants.

\section{Suspensions de procédure et rétractation de témoignage}

En termes de procédure, deux épisodes liés à la sécurité des témoins et où le procureur a été contesté par les chambres concernant l'équité ont momentanément provoqué la suspension du procès. Dans le premier cas (entre juillet 2008 et janvier 2009), l'accès aux documents et leur confidentialité - exigée par l'ONU pour protéger ses sources - étaient en cause. Dans le second cas (entre juillet et octobre 2010), c'est le maintien de l'anonymat d'un intermédiaire accusé par la Défense de falsification de témoignage et de manipulation des témoins qui a posé problème (selon le procureur, cet anonymat devait être maintenu par l'accusation tant que les dispositions assurant la sécurité du témoin ne seraient pas mises en place). Selon certaines ONG présentes en Ituri, ces épisodes ont non seulement considérablement retardé le procès, mais également semé inquiétude et incompréhension dans la région parmi la population, y compris chez les témoins et les victimes (Bueno, 2010).

Autre fait marquant : lors de la troisième audience du procès le 28 janvier 2009, après avoir raconté à la CPI comment il avait été recruté par les soldats des Forces Patriotiques pour la Libération du Congo (FPLC), le premier témoin de l'accusation s'est rétracté. Deux hypothèses ont été émises sur les causes de cette rétractation : (1) des menaces contre sa propre sécurité ou celle de sa famille (pourtant le père lui-même témoignera le lendemain); (2) le risque d'être poursuivi en RDC pour des crimes qu'il avouerait avoir commis pendant qu'il était membre des FPLC. Selon les acteurs de protection de l'enfance, c'est la confrontation de l'enfant à visage découvert face à Thomas Lubanga qui l'a fait se rétracter. Selon le juge Fulford, " [le témoin n'était] pas dans un état approprié pour gérer la pression entourant son témoignage » (Jeune Afrique, 2009).

\section{Les enjeux}

Selon Paolina Massidda, l'une des représentantes des 93 victimes autorisées à participer au procès, ce procès devrait permettre aux victimes de demander réparation, mais aussi de "se reconstruire en tant qu'adultes alors même que leur enfance leur a été volée »(Ballong, 2009). Pourtant nombreuses sont les victimes en Ituri qui préfèrent ne pas aller en justice.

Pour les ONG et l'ONU, il s'agit de poursuivre le plaidoyer contre le recrutement et l'utilisation d'enfants dans les forces et groupes armés, de faire "reconnaître " leur vision de la situation, mais aussi de protéger les victimes et de prévenir d'autres recrutements. "L'ONG Human Rights Watch estime que l'affaire Lubanga a déjà eu un impact sur le comportement des chefs de guerres. Ils ont maintenant pleinement conscience de pouvoir faire eux-

\footnotetext{
2 Pour plus de précisions sur l'affaire, se référer au site de la CPI http://www.icc-

cpi.int/Menus/ICC/Situations+and+Cases/Situations/Situation+ICC+0104/Related+Cases/ICC+0104+0106/Democratic+Republic+of+the+Congo.h tm, ainsi qu'au « portail juridique de La Hague » http://french.lubangatrial.org/
} 
mêmes l'objet de poursuites. " (Ballong, 2009). Selon Wairagala Wakabi (2010a), "Quelle que soit la façon dont se terminera le procès de Thomas Lubanga à la Cour pénale internationale, il aura incontestablement contribué à propulser sous les projecteurs du monde le phénomène des enfants soldats et la détresse qu'ils vivent. »

Pour la CPI, il s'agit de faire un procès exemplaire sur la question du recrutement d'enfants, mais aussi de mettre en place de nouvelles pratiques, en introduisant notamment une nouvelle stratégie en relation avec les victimes:

A key feature of the system established in the Rome Statute is the recognition that the ICC has not only a punitive but also a restorative function. It reflects growing international consensus that participation and reparations play an important role in achieving justice for victims. (CPI, 2006b, § 3)

Pour le procureur de la CPI, Luis Moreno-Ocampo,

cette affaire représente une avancée considérable dans la lutte contre ces crimes graves à l'encontre des enfants. La conscription d'enfants détruit la vie et l'avenir de milliers d'enfants dans le monde. Cette affaire contribuera à dénoncer ce problème et à enrayer ces pratiques criminelles. (CPI, 2006b)

Pour l'accusé et sa défense, il s'agit de démontrer d'une part que les enfants témoins sont manipulés par l'accusation, d'autre part que ceux qui ont participé avaient plus de 15 ans et l'ont fait volontairement. Enfin, que Thomas Lubanga aurait entamé des négociations avec la Mission des Nations Unies en RDC (MONUC) pour une éventuelle démobilisation de ces enfants.

\section{Les dilemmes}

Le premier dilemme concerne la sécurité et l'intégrité des victimes/témoins. Si les témoignages permettent de réduire les chances de recrutement en combattant l'impunité, en témoignant, les enfants et leurs familles s'exposent aux conditions difficiles du procès et au danger de représailles. Le défi est de conjuguer application de la justice et respect de leur dignité et de leur sécurité dans une région encore en guerre où même les membres des FARDC censées défendre la population sont accusées de recrutement, de viol et de pillage. Comme le soulignait un rapport d'évaluation de la protection des témoins par le Tribunal Spécial de la Sierra Leone (Charters, Horn et Vahidi, 2008), "Witnesses are essential to the success of tribunals such as the SCSL, and the quality of their protection and support is vital. If the quality is low, it follows that the quality of the testimony that the witness is able to give may suffer ». Mais jusqu'à quel point la garantie de leur sécurité est-elle possible? Si un procès ne peut plus être poursuivi, faute de capacité à protéger les victimes, à qui sert la justice internationale? En ce sens, «les victimes demandent que l'excès de justice ne soit pas de l'injustice », déclarait Luc Walleyn, un avocat des victimes, à l'occasion de la suspension du procès en juin 2008 (Ben Rhouma, 2008).

Le deuxième dilemme concerne la question cruciale de l'"agencéité " des enfants, autour de figures de l'enfance où s'opposent vulnérabilité et dangerosité, discernement et immaturité. D’abord, au sein même du procès: les enfants sont-ils en mesure de témoigner, sont-ils manipulés? Ensuite, en ce qui concerne leur participation aux groupes armés : le recrutement était-il volontaire ou forcé? Peuvent-ils être tenus responsables des actes commis pendant leur association aux forces et groupes armés et être condamnables pour ces derniers? À cela, les acteurs de protection de l'enfance répondent que les enfants peuvent témoigner (ils ont le discernement suffisant pour ce faire) mais dans des circonstances particulières (préparation, soutien, absence de pressions donc par exemple à visage caché); que les recrutements dits «volontaires " sont le résultat de pressions diverses (donc que les enfants n'avaient pas le discernement suffisant pour être qualifiés de volontaires); enfin, qu'ils peuvent être traduits en justice (ils ont un degré de responsabilité), mais dans les conditions particulières appliquées aux mineurs (en prenant en compte le degré de maturité et en proposant réhabilitation plutôt que répression). Mais si ces spécificités reconnaissent un degré moindre de discernement et de responsabilité, que valent leur participation et leur témoignage?

La défense veut démontrer que les témoins ont été encouragés à mentir sur un certain nombre de points très spécifiques, en particulier ce qui a trait à leur nom, le nom de leurs parents, les écoles qu'ils prétendent avoir fréquentées, et que cela a été fait afin de rendre plus difficile toute vérification des informations les concernant. (Wakabi, 2010b) 
Le troisième dilemme concerne l'universalité des droits humains enchâssée dans des enjeux à caractère politique. Le contexte géopolitique de la RDC en 2006 marque en effet ce procès qui s'inscrit dans une tension entre volonté de justice et paix nationale. 2006 est l'année des premières élections démocratiques depuis la guerre civile, mettant en liesse différents chefs de guerre potentiellement responsables d'autant d'atrocités que les accusés de la CPI. Pourtant, malgré une paix relative dans la majorité du pays, les combats, recrutements, viols et autres atrocités n’ont pas cessé en Ituri, ni dans les Kivus, autour de conflits ethniques, territoriaux et miniers (Montbrial et Moreau Defarges, 2008). Ce procès est donc censé marquer une volonté de justice, mais la nécessité d'une cohésion nationale a conduit la CPI en RDC à se concentrer uniquement sur l'Ituri. Le procès contre Thomas Lubanga est considéré par la population de la région comme injuste pour cette raison. Par ailleurs, le recrutement et l'utilisation d'enfants de moins de 15 ans, s'ils sont considérés par une partie des Congolais comme des faits répréhensibles, ne constituent pas le même "intolérable » que pour les occidentaux. Le choix de cette pratique comme objet du premier procès de la CPI (plutôt que le viol ou le meurtre de civils par exemple), s'il est révélateur pour le procureur d'une volonté de marquer l'importance universelle de protéger les enfants, laisse aux populations le sentiment d'une manipulation et de règlements de comptes externes à leurs préoccupations. La protection des enfants serait un prétexte. Les conséquences pour les acteurs de protection sont variées. Certains groupes armés n'acceptent plus de négocier la sortie d'enfants de leurs rangs par peur d'être dénoncés par les mêmes acteurs avec lesquels ils négocient, et ils abandonnent les enfants avant de passer par la procédure d'intégration aux FARDC. De plus, la longueur et les événements chaotiques de ce procès en décrédibilisent la valeur pour la population.

\section{Mécanisme de surveillance et de communication de l'information sur les graves violations contre les enfants en situation de conflit armé}

Depuis 1996 et le rapport de Graça Machel, les organisations de protection de l'enfance se sont attachées à dénoncer devant la communauté internationale les violations envers les enfants en situation de conflit armé. Mais faute d'une démarche coordonnée et systématique, ces dénonciations sont longtemps restées sans effet. Face à cette situation, la Résolution n 1539 sur les enfants et les conflits armés adoptée par le Conseil de sécurité des Nations Unies en 2004 (Nations Unies, 2004) demandait à son Secrétaire général l'établissement d'un « mécanisme de surveillance et communication de l'information » qui rassemble des informations opportunes, précises et objectives et permette de systématiquement déclencher des réponses appropriées au sujet de six «violations graves » des droits de l'enfant dans les situations de conflit armé : mise à mort ou mutilation, viol ou autres violences sexuelles, recrutement ou utilisation dans les conflits, enlèvements, attaques sur écoles/hôpitaux et enfin refus d'un accès humanitaire.

\section{De la surveillance à l'enlistement..}

En 2005, la Résolution n 1612 du Conseil de sécurité (Nations Unies, 2005) rappelait la n¹539 et exigeait que les parties au conflit qui enrôlaient ou utilisaient des enfants soient "listées », c'est-à-dire mentionnées dans les annexes des rapports sur les enfants et les conflits armés du Secrétaire général au Conseil de sécurité. En 2009, la Résolution $n^{\circ} 1882$ étendait cet «enlistement » aux "parties à un conflit armé qui, en violation du droit international applicable, commettent systématiquement des meurtres et mutilations d'enfants et/ou des viols et autres formes de violence sexuelle contre les enfants, dans des situations de conflit armé ». (Nations Unies, 2009, $\S 3$, p. 2)

\section{... au plan d'action des gouvernements...}

Les Résolutions exigent, en outre, que les parties inscrites élaborent et appliquent des plans d'action détaillant les temps et les étapes concrètes pour mettre fin aux violations et qu'elles agissent en réponse à tous les autres abus et violations commis contre des enfants (Nations Unies, 2004, 2005, 2009). Chaque plan d'action, conçu et adapté par l'ONU et les pays concernés en consultation avec les parties prenantes individuellement, prévoit une vérification indépendante par le Groupe de travail de I'ONU; la plupart des plans prévoient aussi une aide de l'ONU pour la mise en œuvre du plan. 


\section{... et aux sanctions}

Lorsqu'aucun progrès n'est constaté, la Résolution $n^{\circ} 1612$ prévoit des recommandations au Comité de sanctions de I'ONU, comme l'appel à l'arrêt de soutien militaire aux parties en conflit. En cas d'échec du dialogue et du plaidoyer, la Résolution $n^{\circ} 1882$ demande aux États membres d'agir de manière décisive et immédiate contre les criminels persistants de violations contre des enfants, par des mécanismes de justice applicables, nationaux ou internationaux.

\section{En RDC}

En RDC, un mécanisme de surveillance et de communication de l'information est en place depuis 2005, mais malgré l'enlistement des groupes armés et des FARDC, aucun plan d'action n'a encore été signé. Le gouvernement reconnaît avec difficulté que ses propres forces armées recrutent et utilisent des enfants, et ce n'est que sous la menace de sanctions du Conseil de sécurité qu'il dit vouloir prendre des mesures appropriées.

\section{Enjeux et dilemmes}

Selon le schéma idéal, la protection des enfants repose sur l'établissement d'un mécanisme de surveillance de la situation, sur l'engagement au dialogue, sur le plaidoyer et sur le renforcement des dispositifs nationaux de protection des enfants et de responsabilisation pénale des criminels. Les plans d'action devraient fournir la structure du renforcement de la protection des enfants sur le terrain, et les rapports au Conseil de Sécurité de I'ONU un levier d'influence sur les décideurs. Mais le mécanisme est loin d'être fluide et doit surmonter un certain nombre de tensions.

Encore une fois, le recueil de témoignages d'enfants pose des problèmes de sécurité, et la protection des informateurs et des sources demeure essentielle. Or les organisations qui sont en mesure de recueillir les témoignages sont celles qui sont amenées à collaborer d'une manière ou d'une autre avec les forces et groupes armés, ne serait-ce que pour négocier la libération des enfants et réduire les maltraitances. II est donc essentiel qu'elles ne soient pas mises en porte à faux. Malgré certaines mesures de confidentialité offertes par le mécanisme, certaines ne souhaitent pas s'exposer à ce risque.

Par ailleurs, ce mécanisme propose à la fois de dénoncer un gouvernement et d'engager avec lui une politique de protection des enfants, ce qui met les acteurs qui ont signé un protocole d'accord avec les gouvernements dans une position paradoxale de collaboration et de dénonciation. Les témoignages des enfants risquent alors d'être singulièrement polis pour être « entendables».

\section{Visite/échange Nord-Sud}

Les organisations de protection des enfants agissant en RDC tirent leurs ressources financières de leurs partenaires du Nord qui s'attachent à collecter des fonds et mobiliser l'opinion publique sur les situations auxquelles les enfants font face. Le témoignage des enfants soldats congolais fait partie des outils de sensibilisation. C'est dans ce cadre qu'en juin 2004, un groupe d'adolescents français - accompagné de journalistes de presse écrite et audiovisuelle - vient rencontrer durant deux semaines des groupes d'« ex-enfants soldats » de RDC pris en charge par des organisations de protection de l'enfance.

À la demande des acteurs de protection en RDC, l'activité est organisée sous forme d'échange plutôt que d'une « visite » qui mettrait les jeunes Congolais en situation passive. Un certain nombre d'activités sportives, théâtrales, musicales sont organisées. Tous semblent satisfaits du séjour, avec de réels moments d'échange entre les différents groupes d'enfants et de jeunes, tant à Goma qu'à Kinshasa. Entre la presse et les agents de protection de l'enfance en RDC, les rapports sont plus tendus. Les journalistes souhaitent pouvoir montrer les visages des enfants et des jeunes pour rendre la rencontre plus "réelle" et transmissible, alors que les acteurs de protection recommandent l'anonymat des enfants (avec absence ou floutage des visages et transformation des contextes).

Après le séjour, un reportage vidéo, des interviews radio, des articles de presse et de magazine seront présentés en France, de manière complète et documentée, exposant les réalités des ex-enfants-soldats, leurs expériences et 
projets. Mais les visages des enfants congolais seront clairement montrés et accompagnés des prénoms et des lieux de résidence, ce qui représente un risque leur sécurité. De plus, malgré le sentiment des jeunes Français d'avoir vécu un échange et une rencontre qu'ils qualifient d' "inoubliable », aucune information ni aucune copie des émissions de radio et de télé, des vidéos ou des articles de magazines et de journaux ne sont envoyées en retour en RDC, ni à l'organisation ni aux groupes de jeunes et d'enfants.

\section{Les enjeux annoncés}

Pour l'organisation en France, il s'agit de faire connaître le problème, de se faire connaître, et de récolter des fonds pour renforcer le plaidoyer contre le recrutement des enfants et pour financer des projets en RDC. Pour l'organisation en RDC, il s'agit de permettre aux enfants et aux adolescents de rencontrer des jeunes avec une autre réalité, de faire connaître leur histoire tout en assurant leur protection et de récolter des fonds auprès de l'organisation française. Pour les jeunes Français, il s'agit de rencontrer des enfants soldats pour mieux connaître leur expérience, et de la faire connaître à leur entourage. Pour les jeunes Congolais, c'est aussi l'occasion de connaître une autre réalité, d'établir des liens, de partager son expérience.

\section{Rencontre ou prédation?}

Malgré ces enjeux annoncés d'où tous sortiraient "gagnants », la rencontre désirée laisse derrière elle le goût d'une " prédation ", où l'image et l'histoire des enfants congolais auraient été captées pour être exposées, mais où leur réalité " existentielle » n'est plus: les contacts sont rompus. Les enfants et jeunes congolais n'existent plus qu'au travers de leur témoignage. Tout s'est passé comme s'il n'y avait pas eu de rencontre, mais une simple vérification de la figure qui devait faire l'objet de la rencontre, celle de l'enfant-soldat. Le non-respect des règles élémentaires de protection des jeunes Congolais contre toute action de représailles (par exemple en floutant leur visage et en transformant leurs prénoms et provenances) illustre que ces enfants sont devenus des figures désincarnées (une figure ne risque pas d'être abusée, maltraitée, violentée, car elle n'est pas). Finalement, on peut se demander qui témoigne. Il semble que les véritables témoins, ceux qui ont la parole, ne sont pas les ex-enfants soldats, mais les enfants et les journalistes du Nord qui font savoir qu'ils ont rencontré des enfants soldats, comme des images célèbres et fascinantes.

$\mathrm{Au}$ vu de cette expérience pour le moins troublante, peut-on conclure que l'expérience de la rencontre interculturelle est impossible? Ou que le témoignage est inapte à la restituer? Cette situation révèle-t-elle une facette de l'action humanitaire de protection de l'enfance qui repose sur un certain nombre de représentations et figures de l'enfance, certes déviantes, mais fascinantes?

\section{Témoignages " de proximité "}

Dans une tout autre dimension et perspective, les témoignages des enfants sont aussi utilisés auprès de leurs pairs et au sein de leurs communautés. Voici par exemple l'expérience de deux organisations, une en RDC, l'autre en Ouganda, qui travaillent avec des jeunes filles anciennement associées aux forces et groupes armés, victimes de violences sexuelles et/ou prostituées et qui ont des difficultés de réintégration.

Pour ces jeunes filles, dans un premier temps, en situation individuelle ou de groupe restreint avec des encadrants, raconter leur histoire est un moyen privilégié de revenir sur la situation passée et de lui « donner sens » pour se reconstruire. En général, il s'agit de reconstituer les éléments de leur histoire, de décrire leurs émotions dans un milieu où elles se sentent en sécurité, de revenir sur les circonstances de leur enrôlement ou enlèvement, mais aussi de se situer dans le sens que ce dernier prend, car le processus de démobilisation ou de réinsertion transforme totalement l'environnement des enfants et le sens de leur expérience. II arrive par exemple que des jeunes filles anciennement associées à une force armée ou un groupe armé ayant subi des violences sexuelles (au regard des droits humains) ne s'identifient pas comme victimes de violences sexuelles, considérant que leur situation était la situation "normale " d'une femme de commandant (au regard de la culture militaire dans laquelle elles vivaient). Un certain temps après leur sortie des groupes armés et leur arrivée dans un centre de protection, à la suite d'échanges avec leurs pairs, certaines d'entre elles "requalifient » leur expérience comme 
une violence et s'identifient comme victimes ou « survivantes » de violences sexuelles selon le terme anglo-saxon (survivors).

Depuis 2005, dans la région de l'Ituri en RDC, avec le soutien de leurs éducateurs, un groupe de jeunes filles a décidé de créer et de présenter une pièce de théâtre aux communautés environnantes sur cette situation, pour sensibiliser et transformer les pratiques. Et sous un mode voisin, dans le Nord Ouganda, des jeunes filles anciennement enlevées par la Lord's Resistance Army (LRA) se sont regroupées avec pour objectif, grâce à leur témoignage, de sensibiliser les communautés environnantes et de soutenir leurs pairs.

Dans les deux cas, elles mettent d'abord l'accent sur la qualification de leur situation comme une situation de victimes, pour être reconnues comme telles, soutenues et réintégrées (car les communautés les rejettent les considérant comme coupables et devenues impures, "souillées "). De plus, leurs témoignages soulignent leurs autres qualités, leurs capacités de résilience. Enfin, en s'appropriant elles-mêmes leur histoire pour " soutenir leur cause " et celle de leurs pairs, ces jeunes filles se positionnent non plus en victimes passives, mais en actrices de leur situation, dans leur environnement.

\section{Rôles et objectifs}

Les jeunes filles ont un double objectif : dans un premier temps, être reconnues comme victimes pour bénéficier de la clémence de leur communauté et pour souligner le partage des responsabilités sur la question à l'intérieur des communautés; dans un second temps, sortir de leur position de victimes pour "prendre en main " leur sort et " prendre part » à la vie communautaire. Le choix de témoigner pour sensibiliser et soutenir d'autres jeunes filles ayant vécu la même situation semble être, dans leur cas, le moyen de concilier ces deux objectifs.

En assurant leur protection et en soutenant les filles dans leur processus de réintégration, les acteurs de protection, par leur écoute, les échanges qu'ils suscitent et les informations qu'ils fournissent, servent de catalyseurs pour donner un sens nouveau aux histoires respectives. Dans un second temps, ils donnent l'opportunité aux jeunes filles d'utiliser leur histoire pour jouer un rôle dans leur communauté, ils accompagnent leur passage de la position de victime à celle d'actrice. La parole de témoignage, qui servait à raconter une expérience passée, se transforme en parole d'échange, qui permet de prendre part, pour devenir ensuite parole de décision.

\section{D'une figure à l'autre}

Contrairement à la situation précédente où les enfants étaient figés dans leurs rôles fascinants d'ex-soldats et où l'échange entre les deux groupes ne se réalisait pas, ces expériences mettent en évidence la possibilité de passage d'une figure à l'autre (de la coupable à la victime, puis au sujet/actrice), où le catalyseur pourrait bien être la réalité des échanges et la prise de parole au sein des groupes de pairs ou avec des groupes extérieurs. La différence provient aussi de la nature des objectifs des différents acteurs impliqués et des positions que chacun peut prendre dans cette rencontre.

En cela, la position des acteurs de protection de l'enfance vis-à-vis des enfants et des groupes communautaires auxquels ils appartiennent est fondamentale. Même en étant pour la plupart d'entre eux d'une culture différente de celle des enfants (ne serait-ce que du fait de leur origine sociale souvent différente), en donnant l'opportunité aux jeunes filles de prendre une parole allant au-delà de leur position de victime, ils peuvent ouvrir un chemin de réintégration. Mais pour cela on peut faire l'hypothèse qu'il leur faut acquérir eux-mêmes une vision différente des enfants avec lesquels ils travaillent, qui passent de victimes à qui on offre un service à acteurs ou actrices doté(e)s d'une puissance d'agir qu'il s'agit de soutenir. 


\section{En conclusion}

L'exploration de ces différentes formes d'utilisation des témoignages des enfants pour leur protection nous permet de souligner des dilemmes propres à l'intervention humanitaire de protection de l'enfance (pas simplement en $\mathrm{RDC}$ ), dilemmes qui sont issus notamment du contexte interculturel dans lequel cette protection s'inscrit, autour des figures de l'enfance.

On remarque en premier lieu l'aspect éminemment politique dans lequel s'inscrit le phénomène, la vulnérabilité des enfants justifiant les interventions internationales. Lorsque la justice internationale ou le Conseil de sécurité des Nations-Unies dénoncent le recrutement et l'utilisation d'enfants par des forces et groupes armés, les interventions de protection des enfants trouvent une justification officielle. Mais ces interventions risquent d'être compromises par leur inscription politique et de ce fait biaisées par une vision taxée d'occidentale et non appropriée. Les acteurs de protection se trouvent coincés dans une double position d'accusation des gouvernements et de collaboration avec eux, et leurs visions de l'enfance demeurent figées par le caractère intolérable du phénomène consacré par la justice internationale.

En second lieu ressortent de cette exploration les différentes figures de l'enfance qui traversent toute intervention de protection dans ce cadre. En effet, si la protection des enfants trouve sa justification dans la figure d'enfants comme victimes souffrantes, pour participer à la reconstruction et la réinsertion de ces mêmes enfants, il faut leur reconnaître une puissance d'agir, c'est-à-dire aussi une certaine forme de responsabilité, à la fois dans le phénomène de recrutement et dans les actes commis contre la population. Rappelons qu'une partie des enfants s'enrôlent volontairement pour des raisons variées - pour les opportunités économiques, par idéologie ou par besoin de protection - et qu'ils peuvent commettre des actes difficilement pardonnables par les communautés. Dans la négociation pour que les communautés acceptent un retour de ces enfants, leur témoignage peut servir de catalyseur, d'une part pour faire connaître les conditions de leur recrutement et leur caractère de victimes, d'autre part pour montrer leur puissance d'agir de manière positive. L'exemple des jeunes mères célibataires illustre ce que peuvent être les différentes étapes d'une "réhabilitation/guérison ", lorsqu'elles passent par une reconnaissance du statut de victime (pour pouvoir bénéficier du pardon et d'un soutien) pour évoluer vers une "réintégration » à part entière au sein de leur groupe (famille, communauté, société).

Enfin, la question centrale de l'interculturalité se trouve posée. D’une part, autour de la possibilité ou l'impossibilité d'une rencontre, comme dans le cas de la visite du groupe d'enfants français en RDC, lorsque les représentations prennent la place sur l'échange. D'autre part, autour de l'universalité de la CDE, qui d'un côté se base sur une figure d'enfants vulnérables et de l'autre mentionne la nécessité de participation active des enfants, ces visions n'étant pas toujours partagées localement, où l'enfant est considéré comme responsable des actes qu'il commet et où la soumission aux aînés constitue la règle.

Lorsque l'enjeu des acteurs de protection est la réintégration des enfants dans leurs communautés, la question des représentations et leur "négociation » avec les communautés est centrale, en cela que les enfants sortant du monde militaire auront à se conformer à une ou plusieurs de ces représentations pour se réinsérer.

\section{Références bibliographiques}

Agence France Presse. (2009, 28 janvier). Procès Lubanga: pression sur les témoins, le premier se rétracte. Jeune Afrique [En ligne]. http://www.jeuneafrique.com/Article/DEPAFP20090129T131856Z/-CPI-Thomas-LubangaIturi-Proces-Lubanga--pression-sur-les-temoins-le-premier-se-retracte.html (page consultée le 20 avril 2010).

Arzoumanian, N. et Pizzutelli, F. (2003). Victimes et bourreaux : questions de responsabilités liées à la problématique des enfants-soldats en Afrique. Revue internationale de la Croix Rouge, 852, 827-856.

Ballong, S. (2009, 26 janvier). Thomas Lubanga répond de crimes de guerre devant la CPI. Afrik.com [En ligne]. http://www.afrik.com/article16148.html (page consultée le 20 avril 2010).

Ben Rhouma, H. (2008, 3 juillet). Thomas Lubanga pourrait échapper à la justice internationale. Afrik.com [En ligne]. http://www.afrik.com/article14684.html (page consultée le 20 avril 2010). 
Bueno, O. (2010, 13 octobre). Reprise du procès de Lubanga: réactions de l'Ituri. Le procès de Lubanga à la CPI [En ligne]. http://french.lubangatrial.org/2010/10/13/reprise-du-proces-de-lubanga-reactions-deI\%E2\%80\%99ituri/ (page consultée le 18 octobre 2010).

Charters, S., Horn, R. et Vahidy, S. (2008). Best-practice recommendations for the protection and support of witnesses. Free Town : Special Court of Sierra Leone.

Cour pénale internationale. (2006a). Fiche d'information sur l'affaire le Procureur contre Thomas Lubanga Dyilo. Affaire $n^{\circ}$ ICC-01/04-01/06 [En ligne]. http://www.icccpi.int/menus/icc/situations\%20and\%20cases/ situations/situation\%20icc\%200104/related\%20cases/icc\%200104\%200106/democratic\%20republic\%20of\% 20the\%20congo?lan=fr-FR

Cour pénale internationale. (2006b). L'utilisation des enfants soldats au cœur des accusations dans la première affaire de la cour pénale internationale [En ligne]. http://www.icccpi.int/menus/icc/press\%20and\%20media/press\%20releases/2006/child\%20soldier\%20charges\%20in\%20th e\%20first\%20international\%20criminal\%20court\%20case (page consultée le 28 août 2006).

Cour pénale internationale. (2009). Rapport de la Cour sur la stratégie concernant les victimes. Huitième session. La Haye : Cour pénale internationale.

Honwana, A. (2000). Innocents et coupables : les enfants soldats comme acteurs tactiques. Politique africaine, 80, 58-78.

Lee, A.-J. (2009). Understanding and addressing the phenomenon of 'child soldiers': the gap between the global humanitarian discourse and the local understandings and experiences of young people's military recruitment. Oxford : Refugee Studies Centre/University of Oxford (Working Paper $n^{\circ} 52$ ).

Les Principes de Paris (2007). Principes et lignes directrices sur les enfants associés aux forces et groupes armés [En ligne]. Récupéré sur le site de l’Agence des Nations Unies pour les réfugiés à http://www.unhcr.org/refworld/docid/49997af21a.html

Machel, G. (1996). Impact des conflits armés sur les enfants. Rapport de l'expert Graça Machel au Secrétaire Général des Nations Unies en application de la résolution 48/157.

Montbrial, T. et Moreau Defarges, P. (2008). RAMSES 2009 - Turbulences économiques et géopolitique planétaire. Paris : Dunod.

Nations Unies (1989). Convention relative aux droits de l'enfant.

Nations Unies (2004). Les enfants et les conflits armés. Résolution 1539 (2004) adoptée par le Conseil de Sécurité à sa $4948^{\mathrm{e}}$ séance, le 22 avril 2004.

Nations Unies (2005). Les enfants et les conflits armés. Résolution 1612 (2005) adoptée par le Conseil de Sécurité à sa $5235^{\mathrm{e}}$ séance, le 26 juillet 2005.

Nations Unies (2009). Les enfants et les conflits armés. Résolution 1882 (2009) adoptée par le Conseil de sécurité à sa $6176^{\mathrm{e}}$ séance, le 4 août 2009.

Saillant, F. (2011). Droits humains et témoignages : l'épreuve de la culture. Alterstice, 1(2), 3-8.

Wakabi, W. (2010a, 27 janvier). Les remarques préliminaires de la défense de Lubanga - les témoins ont menti, Lubanga n'est pas coupable. Le procès de Lubanga à la CPI [En ligne]. http://french.lubangatrial.org/2010/01/27/les-remarques-preliminaires-de-la-defense-de-lubanga\%E2\%80\%93-les-temoins-ont-menti-lubanga-n\%E2\%80\%99est-pas-coupable/ (page consultée le 20 avril 2010).

Wakabi, W. (2010b, 5 octobre). Le procès de Lubanga jette la lumière sur la détresse des enfants soldats. Le procès de Lubanga à la CPI [En ligne]. http://french.lubangatrial.org/2010/10/05/le-proces-de-lubanga-jette-lalumiere-sur-la-detresse-des-enfants-soldats/ (page consultée le 18 octobre 2010). 